\title{
Flies from a tertiary hospital in Rwanda carry multidrug-resistant Gram-negative pathogens including extended-spectrum beta-lactamase-producing $E$. coli sequence type 131
}

\author{
Stefan E. Heiden ${ }^{1}$, Mathis S. E. Kurz ${ }^{2}$, Jürgen Bohnert ${ }^{3}$, Claude Bayingana ${ }^{4}$, Jules M. Ndoli ${ }^{4,5}$, Augustin Sendegeya ${ }^{4,5}$, \\ Jean Bosco Gahutu ${ }^{4,5}$, Elias Eger ${ }^{1}$, Frank P. Mockenhaupt ${ }^{2}$ and Katharina Schaufler ${ }^{1 *}$ (D)
}

\begin{abstract}
Multidrug-resistant gram-negative (MRGN) bacteria are a serious threat to global health. We used genomics to study MRGN obtained from houseflies in a tertiary Rwandan hospital. Our analysis revealed a high abundance of different MRGN including E. coli pathogenic lineage ST131 suggesting the important role of flies in disseminating highly virulent pathogens in clinical settings and beyond.
\end{abstract}

Keywords: MRGN, Vector flies, Virulence

\section{Text}

Multidrug-resistant gram-negative (MRGN) bacteria include Escherichia (E.) coli, Klebsiella spp., Enterobacter (E.) cloacae, Acinetobacter spp., and Pseudomonas (P.) aeruginosa, and others, and cause a variety of severe infections like diarrhea, pneumonia, sepsis, endocarditis and urinary tract infection (UTI). Studies estimate 700.000 fatalities caused by antibiotic-resistant pathogens each year with increasing numbers [1]. In addition to their common occurrence as nosocomial pathogens, MRGN have been frequently found in livestock and the environment. Flies have only recently come into spotlight as carriers of resistant bacteria, and their major route of colonization stems from walking on contaminated surfaces [2]. The detection of antibiotic-resistant E. coli from flies captured in a livestock facility was thus unsurprising [3]. Another study has shown that houseflies from hospitals in the UK carried different bacteria resistant to antibiotics [4]. We investigated if houseflies captured in a tertiary hospital in Rwanda carried

\footnotetext{
* Correspondence: katharina.schaufler@uni-greifswald.de

'Pharmaceutical Microbiology, Institute of Pharmacy, University of Greifswald, Friedrich-Ludwig-Jahn-Str. 17, 17489 Greifswald, Germany

Full list of author information is available at the end of the article
}

clinically relevant MRGN pathogens. In African hospital settings, where hygienic conditions may be suboptimal [5], flies might function as underestimated vectors for the distribution of antibiotic-resistant bacteria.

We examined 42 flies randomly captured in fly traps within 4 weeks in a tertiary hospital in Rwanda in 2014 [5]. Sampling locations included surgery, gynecologic and other wards (Fig. 1a/b). Because we initially focused on cefotaxime-resistant representatives, bacteria carried by flies were first enriched in tryptic soy broth and then cultured on chromogenic agar (CHROMagar-ESBL, Mast Diagnostica, Germany) supplemented with $2 \mu \mathrm{g} /$ $\mathrm{mL}$ cefotaxime. For "extended-spectrum beta-lactamase (ESBL)-positive" colonies, ESBL and/or ampicillinase $(\mathrm{AmpC})$ production was verified (ESBL-AmpC-Detection Test, Mast Diagnostica [6]), and strains positive for AmpC only were excluded. After preselecting putative strains of E. coli, Klebsiella spp., Enterobacter spp., Acinetobacter spp., P. aeruginosa, Citrobacter spp., and Raoultella spp., we confirmed the bacterial species using MALDI-TOF (Bruker Daltonics, Germany). Additional phenotypic resistance screening was performed on the VITEK 2 system (bioMérieux, France) and for colistin resistance on 96-well microtiter plates investigating minimal

(c) The Author(s). 2020 Open Access This article is distributed under the terms of the Creative Commons Attribution 4.0 International License (http://creativecommons.org/licenses/by/4.0/), which permits unrestricted use, distribution, and reproduction in any medium, provided you give appropriate credit to the original author(s) and the source, provide a link to the Creative Commons license, and indicate if changes were made. The Creative Commons Public Domain Dedication waiver (http://creativecommons.org/publicdomain/zero/1.0/) applies to the data made available in this article, unless otherwise stated. 


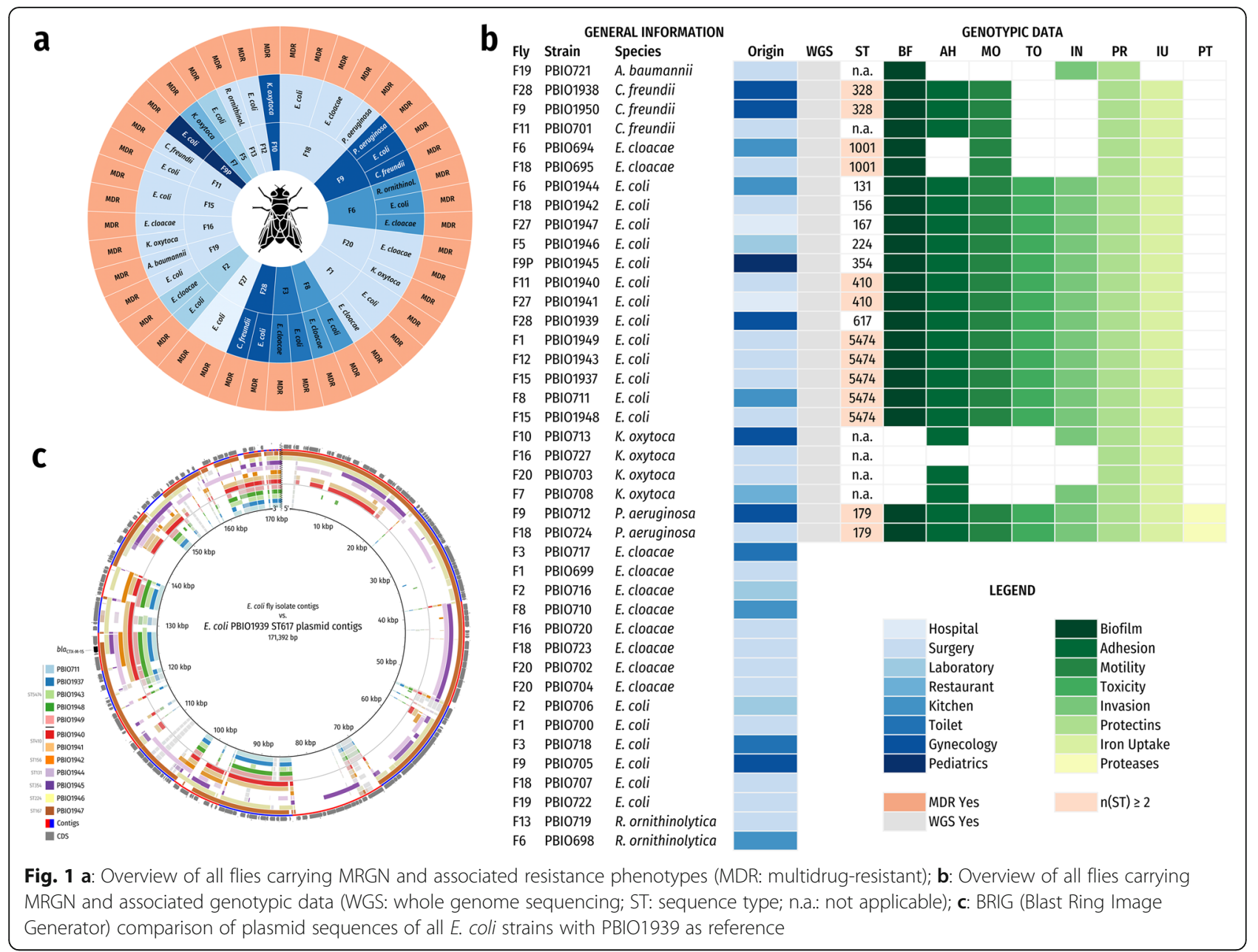

inhibitory concentrations in triplicates. Randomly selected strains (Fig. 1a/b) were whole genome sequenced (WGS) on an Illumina MiSeq/NovaSeq 6000 (Eurofins Genomics Europe Sequencing $\mathrm{GmbH}$, Germany). Raw reads were quality-trimmed, adapter-trimmed and contaminantfiltered using BBDuk from BBTools (https://sourceforge. net/projects/bbmap/files/BBMap_38.41.tar.gz/download). After de-novo assembly using shovill/SPAdes (https:// github.com/tseemann/shovill/archive/v1.0.4.tar.gz; http:// cab.spbu.ru/files/release3.13.1/SPAdes-3.13.1.tar.gz) and Velvet, draft genomes were polished by mapping all trimmed reads back to the contigs with bwa (https:// github.com/lh3/bwa/releases/download/v0.7.17/bwa-0.7.1 7.tar.bz2) and calling variants with Pilon (https://github. com/broadinstitute/pilon/releases/download/v1.23/

pilon-1.23.jar). E. coli plasmid sequences of PBIO711 and PBIO1939 were manually extracted using similarity searches (BLASTn Megablast) against the NCBI nucleotide collection for visualization in BRIG (Blast Ring Image Generator) (https://sourceforge.net/projects/brig/ files/dev/BRIG-0.95-dev.0004.zip/download). Sequence type
(ST), antibiotic resistance/virulence gene and singlenucleotide polymorphism (SNP) detection was carried out using mlst, abricate, and snippy (https://github.com/tseemann/mlst/archive/v2.16.1.tar.gz; https:/github.com/tseemann/abricate/archive/v0.8.11.tar.gz; https://github.com/ tseemann/snippy/archive/v4.4.1.tar.gz). We inferred a core SNP phylogeny for ST5474. Alignments were filtered for recombinations using Gubbins (https:// github.com/sanger-pathogens/gubbins/archive/v2.3.4. tar.gz) and core SNPs extracted using snp-sites (1745 sites; https:/github.com/sanger-pathogens/snp-sites/ archive/v2.4.1.tar.gz). A maximum likelihood tree was inferred with RAxML-NG (https://github.com/amko zlov/raxml-ng/releases/download/0.9.0/raxml-ng_v0.9. 0_linux_x86_64.zip) using GTR + G. The best-scoring maximum likelihood tree was midpoint-rooted and visualized in FigTree (https://github.com/rambaut/figtree/releases/download/v1.4.4/FigTree.v1.4.4.zip).

Overall $48 \%(20 / 42)$ of flies carried antibiotic-resistant bacteria. Thirty-six percent (15/42) carried ESBLproducing E. coli, 19\% (8/42) E. cloacae, 9\% (4/42) K. 
oxytoca, 7\% (3/42) C. freundii, 4\% (2/42) R. ornithinolytica, 4\% (2/42) P. aeruginosa, and 2\% (1/42) A. baumannii. Twelve flies (29\%) carried more than one antibioticresistant bacterial genus of which three (F6, F9 and F18) carried three different pathogens (Fig. 1a/b).

All strains were phenotypically multidrug-resistant and thus termed MRGN (Fig. 1a), however they were not resistant to carbapenems or colistin. WGS revealed carriage of different antimicrobial resistance genes such as bla $a_{\mathrm{CTX}-\mathrm{M}-15}$, aac [3]-IIa, and tet(A)/(B) (Table S1). Eight different STs were observed including ST131 and ST410 (Fig. 1b). Interestingly, these represent international high-risk clonal lineages [7, 8], which combine antimicrobial resistance with highlevel virulence. The ST131 strain harbored ten resistance genes and 31 virulence-associated genes including the pap operon linked to UTI [9] (Table S1).

In addition, we observed five $E$. coli strains of ST5474, which is a ST recently associated with enterotoxigenic E. coli (ETEC) causing diarrhea [10]. This might point towards fly pollution through stoolcontaminated surfaces, possibly through a common source. However, note that we did not detect the ETEC-defining heat-labile and/or heat-stable toxins. Our phylogenetic analysis suggested clonality among our five ST5474 strains (1-9 SNPs/aligned Mbp), and similarity to five publicly available ST5474 genomes (178-560 SNPs/aligned Mbp) (Figure S2).

Three E. coli strains (PBIO1939, PBIO1940 and PBIO1941), which did not only originate from individual flies captured in different wards but belonged to two different clonal lineages (ST410 and ST617), carried similar resistance genes (Table S1), however they differed in their overall plasmid content (Fig. 1c).

The two $P$. aeruginosa genomes contained several previously described virulence features mandatory for severe invasive infections including flagella, the type III secretion system, type IV pili, as well as toxins and proteases. The A. baumannii genome carried virulence genes associated with serum survival and invasion (phospholipase PLC) (Table S1). Overall, all analyzed genomes showed high virulence potentials (Fig. 1b).

Our results demonstrate that half of the flies in this tertiary hospital in Rwanda carried virulent MRGN pathogens including the pathogenic clonal E. coli lineage ST131. High pre-admission and even higher discharge rates at this facility [5] may suggest that a) patients and caregivers were the source of MRGN for the flies and b) that flies play a role in the transmission of antimicrobialresistant pathogens within clinics and in mirroring the burden of antimicrobial resistance [4] at that time. Even though the actual transmission of MRGN bacteria through flies to humans awaits verification, respective modelling results point strongly into this direction [11].

\section{Supplementary information}

Supplementary information accompanies this paper at https://doi.org/10. 1186/s13756-020-0696-y.

Additional file 1: Table S1. Results based on whole genome sequence analysis. Abbreviations: BLA: beta-lactams (incl. ampicillin, piperacillin, cefuroxime, cefpodoxime, cefotaxime, ceftazidime); GEN: gentamicin; CIP: ciprofloxacin/moxifloxacin; SXT: sulfamethoxazole-trimethoprim; TET: tetracycline; ST: sequence type; resistance, virulence and plasmid genes are based on the abricate (https://github.com/tseemann/abricate) abbreviations using the databases Resfinder, ARG-ANNOT, CARD, NCBI Bacterial Antimicrobial Resistance Reference Gene Database,

PlasmidFinder, VFDB, and Ecoli_VF.

Additional file 2: Figure S2. Phylogenomic tree of five $E$. coli sequence type (ST) 5474 fly isolates (strain names colored according to Fig. 1c) and publicaly available WGS data of five ST5474 strains (raw read accession nos.; black).

\section{Abbreviations}

ESBL: Extended-spectrum beta-lactamase; ETEC: Enterotoxigenic E. coli; MRGN: Multidrug-resistant gram-negative; SNP: Single-nucleotide polymorphism; ST: Sequence type; UTI: Urinary tract infection; WGS: Whole genome sequencing

\section{Acknowledgments}

We thank Torsten Semmler (Robert Koch Institute, Berlin, Germany) and Sebastian Guenther (University of Greifswald, Greifswald, Germany) for their support during the initial sequence analysis.

\section{Authors' contributions}

KS and FPM designed and drafted the manuscript. Experiments were performed by SEH, EE and MSEK. JB, CB, JMN, AS and JBG helped analyzing the results. JB, CB, JMN, AS and JBG helped in proofreading and editing of the manuscript. All authors read and approved the final manuscript.

\section{Funding}

Investigations in Rwanda were supported by the German Federal Ministry for Economic Cooperation and Development via the ESTHER programme (Ensemble pour une Solidarité Thérapeutique Hospitalière En Réseau). We acknowledge support for the Article Processing Charge from the DFG (German Research Foundation, 393148499) and the Open Access Publication Fund of the University of Greifswald.

\section{Availability of data and materials}

The data for this study have been deposited in the European Nucleotide Archive (ENA) at EMBL-EBI under accession number PRJEB36565 (https:// www.ebi.ac.uk/ena/data/view/PRJEB36565)

Ethics approval and consent to participate

Not applicable.

Consent for publication

Not applicable.

\section{Competing interests}

The authors declare that they have no competing interests.

\section{Author details}

${ }^{1}$ Pharmaceutical Microbiology, Institute of Pharmacy, University of Greifswald, Friedrich-Ludwig-Jahn-Str. 17, 17489 Greifswald, Germany.

${ }^{2}$ Charité-Universitätsmedizin Berlin, Berlin, Germany. ${ }^{3}$ University Medicine Greifswald, Greifswald, Germany. ${ }^{4}$ University of Rwanda, Kigali, Rwanda.

${ }^{5}$ University Teaching Hospital of Butare, Butare, Rwanda. 
Received: 23 October 2019 Accepted: 7 February 2020

Published online: 17 February 2020

\section{References}

1. Brogan DM, Mossialos E. A critical analysis of the review on antimicrobial resistance report and the infectious disease financing facility. Glob Health. 2016;12:8

2. Khamesipour F, Lankarani KB, Honarvar B, Kwenti TE. A systematic review of human pathogens carried by the housefly (Musca domestica L.). BMC Public Health. 2018;18(1):1049.

3. Alves TD, Lara GHB, ParizMaluta R, Ribeiro MG, Leite DD. Carrier flies of multidrug-resistant Escherichia coli as potential dissemination agent in dairy farm environment. Sci Total Environ. 2018;633:1345-51.

4. Boiocchi F, Davies DP, Hilton AC. An Examination of Flying Insects in Seven Hospitals in the United Kingdom and Carriage of Bacteria by True Flies (Diptera: Calliphoridae, Dolichopodidae, Fanniidae, Muscidae, Phoridae, Psychodidae, Sphaeroceridae). J Med Entomol. 2019;56(6):1684-97.

5. Kurz MSE, Bayingana C, Ndoli JM, Sendegeya A, Durst A, Pfuller R, et al. Intense pre-admission carriage and further acquisition of ESBL-producing Enterobacteriaceae among patients and their caregivers in a tertiary hospital in Rwanda. Tropical Med Int Health. 2017;22(2):210-20.

6. Clinical and Laboratory Standards Institute. Performance Standards for Antimicrobial Disk and Dilution Susceptibility Tests for Bacteria Isolated from Animals-Third Edition: Approved Standard M31-A3. 2008, CLSI, Wayne,PA, USA.

7. Stoesser N, Sheppard AE, Pankhurst L, De Maio N, Moore CE, Sebra R, et al. Evolutionary history of the global emergence of the Escherichia coli epidemic clone ST131. MBio. 2016;7(2):e02162.

8. Schaufler K, Semmler T, Wieler LH, Wohrmann M, Baddam R, Ahmed N, et al. Clonal spread and interspecies transmission of clinically relevant ESBLproducing Escherichia coli of ST410--another successful pandemic clone? FEMS Microbiol Ecol. 2016;92(1):fiv155.

9. Wullt B, Bergsten G, Samuelsson M, Svanborg C. The role of P fimbriae for Escherichia coli establishment and mucosal inflammation in the human urinary tract. Int J Antimicrob Agents. 2002;19(6):522-38.

10. Begum $Y A$, Rydberg HA, Thorell $K$, Kwak $Y K$, Sun $L$, Joffre E, et al. In Situ Analyses Directly in Diarrheal Stool Reveal Large Variations in Bacterial Load and Active Toxin Expression of Enterotoxigenic Escherichia coli and Vibrio cholerae. mSphere. 2018;3(1):e00517.

11. Evers EG, Blaak H, Hamidjaja RA, de Jonge R, Schets FM. A QMRA for the transmission of ESBL-producing Escherichia coli and Campylobacter from poultry farms to humans through flies. Risk Anal. 2016;36(2):215-27.

\section{Publisher's Note}

Springer Nature remains neutral with regard to jurisdictional claims in published maps and institutional affiliations.

Ready to submit your research? Choose BMC and benefit from:

- fast, convenient online submission

- thorough peer review by experienced researchers in your field

- rapid publication on acceptance

- support for research data, including large and complex data types

- gold Open Access which fosters wider collaboration and increased citations

- maximum visibility for your research: over $100 \mathrm{M}$ website views per year

At $\mathrm{BMC}$, research is always in progress.

Learn more biomedcentral.com/submissions 